and illnesses. Notwithstanding the presence of mold in some THUs, the majority of the complaints were linked to the presence of formaldehyde in ambient air within THUs.

The scientific literature confirms that formaldehyde is capable of causing a variety of symptoms and problems, including skin and mucosal irritation, sensitization, decrements in pulmonary function, and the development and aggravation of asthma.

The US Agency for Toxic Substances and Disease Registry (ATSDR) has set a Minimum Risk Level (MRL) for formaldehyde of 8 parts per billion (ppb) for exposure beyond 365 days. The National Institute of Occupational Safety and Health (NIOSH) has established a Recommended Exposure Level (REL) of $16 \mathrm{ppb}$ (time weighted average) for occupational exposure. Formaldehyde also is classified by the International Agency for Research on Cancer (IARC) as carcinogenic to humans (Group I).

Air quality studies of FEMA-supplied THUs measured formaldehyde concentrations between 3.0 to $4,480 \mathrm{ppb}$. Some occupants of THUs were probably exposed for many months to levels of formaldehyde exceeding $100 \mathrm{ppb}$, with excursions up to $500 \mathrm{ppb}$. Many medical symptoms and problems reported by DPs probably were related to formaldehyde exposure while living in THUs.

This unfortunate US experience should be a lesson to other countries that provide THUs to displaced persons following a disaster, or in non-emergency situations. Temporary housing units should be tested for formaldehyde before providing them for human inhabitation.

Keywords: displaced persons; formaldehyde; Hurricane Katrina; symptoms; temporary housing units; toxic

Prehosp Disaster Med

\section{All-Hazards Approach to Emergency Preparedness} Bruria Adini $M A, P b D ;, 1,2$ Avishay Goldberg $M A, M P H, P b D ; 2$

Daniel Laor MD, MHA, $;^{1,2}$ Robert Coben PbD, ${ }^{3}$ Yaron Bar-Dayan $M D, M H A^{2,4}$

1. Emergency and Disaster Management Division, Ministry of Health, Israel

2. PReparED Research Center, Ben-Gurion University of the Negev, Israel

3. Center for Medical Education, Hebrew University, Jerusalem, Israel

4. Meir Medical Center, Israel

Introduction: Medical institutions are required to maintain preparedness to various emergency scenarios, including conventional mass-casualty events (MCEs), chemical or toxicological mass-casualty events (MTEs), and biological events. The aim of this study was to examine the relationship between preparedness to one emergency scenario to the level of preparedness to other emergency scenarios.

Methods: The emergency preparedness levels of all general hospitals were evaluated based on a structured tool that includes measurable parameters. The evaluation encompassed the various elements of emergency preparedness, including standard operating procedures ( $\mathrm{SOP}$ ), infrastructure and equipment, training and drills, and levels of knowledge. The emergency preparedness levels for MCEs, MTEs, and biological events were evaluated and compared.
Results: Moderate correlations were found between the levels of overall preparedness to the various emergency scenarios: MCE; MTE (0.548; 0.006); MCE; biological event (0.541; 0.009); MTEs; biological event (0.458; 0.032). Significant correlations were found between SOPs of MCEs and MTEs $(0.704,0.001)$; between training and drills for all scenarios: MCEs; MTEs (0.626; 0.003), MCEs; biological event $(0.658,0.002)$, and MTE; biological event $(0.586$; 0.008 ); and between infrastructure and equipment for MCEs; MTEs $(0.458 ; 0.032)$. No other significant correlations were found.

Conclusions: The emergency preparedness of hospitals positively correlates with the emergency preparedness to other scenarios, such as MTE and biological events. This relationship does not systematically characterize all elements of emergency preparedness, and is evident especially in the area of training and drills.

Keywords: all-hazards; emergency; preparedness

Prebosp Disaster Med

\section{Confronting Large-Scale Sudden Disasters: Prehospital-In; Hospital-Out \\ Efraim Laor \\ University of Haifa, Haifa, Israel}

Large-scale sudden disasters (LSSDs), due to both natural and technological hazards, are low probability, high-impact events that inflict injury or relocation upon hundreds of thousands or even several millions of people per incident. Many of the affected require medical, physical, and mental treatment.

"Hospitals" are institutions where the real medical treatment is administered. The ambulance services and community-care clinics nicknamed "prehospital" are tasked to provide prompt life-support, stabilization, and transfer of patients- "Scoop and Run"-to a hospital, to be released as soon as possible to "post-hospital", recovery care.

In contrast to ever-ready operational capacity that corresponds to daily emergencies, there is an unbridgeable gap between standing-ops capabilities and what is essential to be done during LSSDs to save lives and reduce damage to health. Instead of hospitals, to call patients for refrain from coming to hospitals- they should extend their proficiency, ethics, personnel, and materiel to "prehospital" systems. In responding to emergencies, laypeople play the decisive role and institutions a secondary one; in preparedness and mitigation, institutions play the decisive role.

As part of preparedness and mitigation measures, hospitals should enhance the participation of every individual, every group, and every community in the emergency management process by improving the methods of coping with perilous circumstances within the family unit and the community.

Hospitals are resourceful and privileged during the preparedness phase, but ineffective during the response phase when it is imperative to call for a shift of duties. Hospitals should contribute to trim Criteria and Standards for the Level of Medical Treatment, one of the realistic methods to augment operational capacity. Only hospitals are capable of granting professional legitimacy to such approach.

Large-scale sudden disasters specifically natural hazard and technological disasters, create dilemmas that crush tra- 